\title{
Red cell distribution width and red cell distribution width to total serum calcium ratio as major predictors of severity and mortality in acute pancreatitis
}

\author{
Marta Gravito-Soares ${ }^{1,2^{*+}}$ (DD, Elisa Gravito-Soares ${ }^{1,2+} \mathbb{D}$, Dário Gomes ${ }^{1,2}$, Nuno Almeida ${ }^{1,2}$ and Luís Tomé $e^{1,2}$
}

\begin{abstract}
Background: Acute pancreatitis (AP) is associated with considerable morbidity and mortality. Current severity scores include multiple variables and some of them are only complete within $48 \mathrm{~h}$ of admission. Red cell distribution width (RDW) is a simple and routine parameter that seems to be related to inflammatory status. Our aims were to evaluate the diagnostic value of RDW in severity and mortality of AP comparing with other prognostic scoring systems.

Methods: Retrospective case-control study of a total of 312 patients with AP admitted between 2014 and 2016. Patients with severe AP (cases) were compared with patients with mild AP (controls) in the 1:1 proportion. Additionally, a comparison between survivor and nonsurvivor AP patients was performed. Diagnosis and severity of AP were defined according to the revised Atlanta classification 2012. Variables evaluated included demographics, comorbidities, hospital stay, laboratorial parameters, arterial blood gas analysis, prognostic scores within $24 \mathrm{~h}$ of admission (Ranson, BISAP and Modified Marshall) and mortality.

Results: Included 91 cases of severe AP, most males (58.2\% vs 51.6\%; $p=0.228$ ) with mean age of $64.8 \pm 16.3$ years (vs 67. $9 \pm 13.7 ; p=0.239)$. RDW on was higher in patients with severe AP (14.6 \pm 1.3 vs $12.7 \pm 0.5 ; p<0.001)$, as well as RDWoh-toserum calcium ratio $(1.8 \pm 0.3$ vs $1.3 \pm 0.1 ; p<0.001)$. After multivariate and ROC curve analysis, RDW oh (AUROC: 0.960 ;

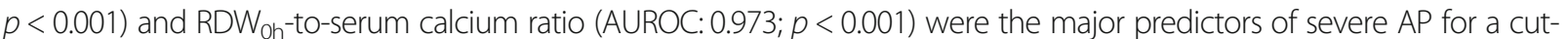
off value of 13.0 (S: 92.7\%; Sp: 84.3\%) and 1.4 (S: 96.3\%; Sp: 84.3\%), respectively. These factors were superior to prognostic scores, such as Ranson (AUROC: 0.777; $p<0.001$; cut-off: 3.0), BISAP (AUROC: 0.732; $p<0.001$; cut-off: 2.0 ) and Modified Marshall (AUROC: 0.756; $p<0.001$; cut-off: 1.0). The mortality rate was 8.8\% (16/182), all cases associated with severe AP

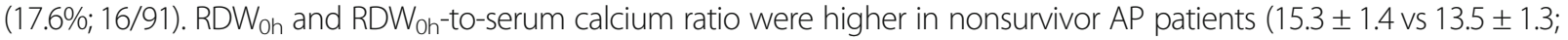
$p<0.001$ and $2.0 \pm 0.3$ vs $1.6 \pm 0.3 ; p<0.001$, respectively). In multivariate and ROC curve analysis, RDW Oh (AUROC: 0.842 ;

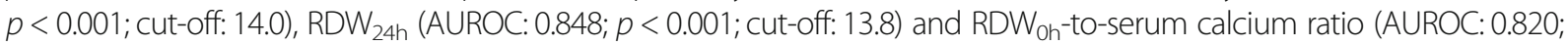
$p<0.001$; cut-off: 1.7) were independent predictors for AP mortality, superior to conventional prognostic scoring systems Ranson (AUROC: 0.640; $p=0.003$; cut-off:3.0), BISAP (AUROC: 0.693; $p=0.017$; cut-off: 2.0) and Modified Marshall (AUROC: 0.806; $p<0.001$; cut-off:1.0).
\end{abstract}

Conclusions: RDW is a simple routine parameter, available at admission. This AP cohort showed that $\mathrm{RDW}_{\mathrm{oh}}>13.0$ and $\mathrm{RDW}_{\text {oh-to-total serum calcium ratio }}>1.4$ were excellent predictors for severity and $\mathrm{RDW}_{\mathrm{Oh}}>14.0$ and $\mathrm{RDW}_{\text {oh }}$-to-total serum calcium ratio $>1.7$ were very-good predictors for mortality, being superior to conventional prognostic scoring systems.

Keywords: Red cell distribution width, Total serum calcium, Acute pancreatitis, Severity, Mortality

\footnotetext{
* Correspondence: ms18498@gmail.com

${ }^{\dagger}$ Marta Gravito-Soares and Elisa Gravito-Soares contributed equally to this work.

'Department of Gastroenterology, Centro Hospitalar e Universitário de

Coimbra, 3000-075 Coimbra, Portugal

${ }^{2}$ Faculty of Medicine, University of Coimbra, 3000-075 Coimbra, Portugal
}

(c) The Author(s). 2018 Open Access This article is distributed under the terms of the Creative Commons Attribution 4.0 International License (http://creativecommons.org/licenses/by/4.0/), which permits unrestricted use, distribution, and reproduction in any medium, provided you give appropriate credit to the original author(s) and the source, provide a link to the Creative Commons license, and indicate if changes were made. The Creative Commons Public Domain Dedication waiver (http://creativecommons.org/publicdomain/zero/1.0/) applies to the data made available in this article, unless otherwise stated. 


\section{Background}

Acute pancreatitis (AP) is an acute inflammation of the pancreatic parenchyma induced by activated pancreatic enzymes due to multiple causes [1,2]. Prognosis of AP depends on its severity, currently classified as mild, moderately severe and severe, according to the revised Atlanta classification (RAC) 2012, which emphasizes the presence of persistent organ failure using the modified Marshall (MM) score [3]. Despite most patients have a mild disease, $20 \%$ of AP patients develop a severe clinical course associated with significant morbidity and mortality (7-42\%) [2]. Early identification of patients at increased risk of severe and fatal AP is crucial to improve prognosis through a prompt medical/ endoscopic treatment and admission to a specialized intensive care unit [4]. An ideal marker/prognostic score should be simple, economic, noninvasive, accurate, and quantitative $[4,5]$. Several prognostic scoring systems and biological markers have been used to predict severity and mortality in AP [2, 5]. However, most of them are complex and not applicable early enough. Ranson and Bedside Index for Severity in AP (BISAP) are specific and widely used prognostic scoring systems due to easy evaluation and availability in the first $24 \mathrm{~h}$ [5]. Single serum markers have also been evaluated, being C-reactive protein (CRP) probably the most useful [4]. However, $\mathrm{CRP} \geq 15 \mathrm{mg} / \mathrm{dL}$ at $48 \mathrm{~h}$ was found to be a predictor of poor prognosis, and therefore a limited severity discriminator in the early phase of AP [5-7]. Until now, no single serum marker is able to predict severity or mortality in AP at admission. Red cell distribution width (RDW) is a routine parameter of the complete blood count test, described as simple, easy, inexpensive and quantitative that measures the size heterogeneity of peripheral red blood cell (RBC), known as anisocytosis [8]. RDW has been associated with inflammatory markers such as CRP, interleukin- 6 and fibrinogen. It revealed to be a good predictor of mortality risk in elderly, critically-ill patients and patients with acute or chronic cardiovascular and respiratory diseases [8-10]. Besides those conditions, RDW has also been evaluated as a mortality predictor in AP [10-13]. However, few recent works showed contradictory results in assessing RDW at admission for AP severity and its relation with well-stablished AP-specific prognostic scores or other serum makers, such as total serum calcium (TSC) [14, 15]. The aims of this study are: (1) to assess RDW ability in predicting AP severity, (2) to assess RDW predictability for AP mortality, and (3) to compare RDW with other serum markers and AP-specific prognostic scoring systems, namely Ranson, BISAP and MM.

\section{Methods}

\section{Study design and patients}

A retrospective case-control study was conducted at the Centro Hospitalar e Universitário de Coimbra from January 2014 to December 2016, including a total of 312 consecutive admissions with AP in our gastroenterology department. Inclusion criteria were age over 18 years-old, a minimum hospital stay of $24 \mathrm{~h}$ and a diagnosis of AP according to the RAC [3] (at least two criteria: typical clinical presentation including acute persistent abdominal pain, serum amylase exceeding 3 times the upper limit of normal (100 IU/L) and characteristic findings on abdominal ultrasonography and/or computed tomography). Exclusion criteria included patients with incomplete data and the presence of underlying factors that could change RDW, such as infectious or immunosuppressive conditions/therapy, active malignancy, chronic use of erythropoietin, recent transfusion history, pregnancy or trauma $[10,16]$. Ninety-one patients with severe AP were identified during the study period (cases), who were compared with patients with mild AP (controls), in the 1:1 proportion. We randomly selected 91 patients from a total of 146 patients with mild AP to incorporate the control group, using Microsoft Excel (Microsoft, Redmon, WA, USA), and therefore avoid statistical bias. AP severity was also defined according to the RAC in severe, based on the presence of single or multiple persistent organ failure $(>48 \mathrm{~h})$ and/or local complications [3]. Organ failure was classified according to MM including cardiovascular, respiratory and renal failures [3]. In addition, patients who died as a result of AP were compared with AP survivor patients (Fig. 1). Variables evaluated included age, gender, AP etiology, organ failure and local/systemic complications AP-associated, comorbidities, smoking habits (more than 10 cigars/day) and alcohol consumption (more than $20 \mathrm{~g}$ alcohol/day). Biochemical and arterial blood gas (ABG) tests at admission were also registered, including white blood cells (WBC) count, aspartate aminotransferase (AST), alanine aminotransferase (ALT), total bilirubin, platelet count, blood urea nitrogen $(\mathrm{BUN})$, creatinine, international normalized ratio (INR), albumin, lactate dehydrogenase (LDH), serum glucose, hemoglobin, hematocrit, serum amylase, $\mathrm{C}$-reactive protein at $0 \mathrm{~h}\left(\mathrm{CRP}_{\mathrm{Oh}}\right)$, $\mathrm{CRP}$ at $24 \mathrm{~h}\left(\mathrm{CRP}_{24 \mathrm{~h}}\right)$, arterial lactate, arterial partial pressure of oxygen, D-dimers, TSC, RDW at $0 \mathrm{~h}$ $\left(\mathrm{RDW}_{0 \mathrm{~h}}\right), \mathrm{RDW}$ at $24 \mathrm{~h}\left(\mathrm{RDW}_{24 \mathrm{~h}}\right)$. Additionally, $\mathrm{RDW}_{0 \mathrm{~h}}$-to-TSC ratio and $\mathrm{RDW}_{0 \mathrm{~h}}$-to-platelets ratio were evaluated. RDW was also compared with validated and widely used AP prognostic scores at first $24 \mathrm{~h}$, including Ranson, BISAP and MM scores. The primary endpoints were severity and in-hospital mortality associated with AP. 


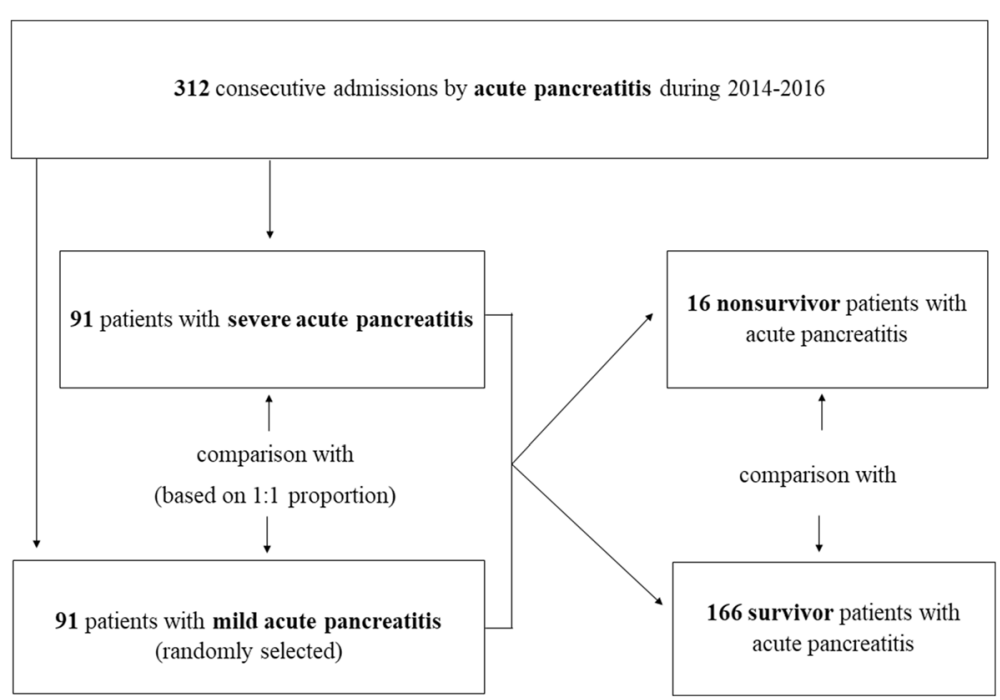

Fig. 1 Flow chart of design study

\section{Statistical analysis}

Statistical analysis was carried out using social package for social sciences version 22.0 for Windows (SPSS Inc., Chicago, IL, USA). The level of significance was set at $p$ value less than 0.05 . Normality of data distribution was assessed with Kolmogorov-Smirnov or Shapiro-Wilk test. Continuous data were expressed as mean and standard deviation (SD) or median and interquartile range based on the normality of distribution. Groups were compared using Student's t-test or Mann-Whitney test. Categorical variables were expressed as frequency and percentage and compared using $\mathrm{X}^{2}$-test or Fisher's exact test. Multivariate logistic regression analysis with the determination of adjusted odds ratio (aOR) and area under the receiver operating characteristic curve (AUROC) were also applied, assigning the best cut-off in terms of sensitivity and specificity.

\section{Results}

\section{Characterization of study population}

A total of 312 eligible patients were admitted with AP during three consecutive years. One-hundred and forty-six patients had mild AP, 75 (24.0\%) moderately severe AP and 91 (29.2\%) severe AP. Of the total of 182 enrolled patients (91 with severe AP and 91 with mild AP after random selection), 54.9\% $(n=100)$ were males with mean age of $66.3 \pm 15.1$ years. Most patients $(n=$ $102 ; 56.0 \%)$ had gall bladder stones. The most common cause of acalculous AP was alcohol $(n=61 ; 76.2 \%)$. An abdominal computed tomography (CT) was performed in $96.7 \%(n=88)$ of patients with severe AP. Among these patients, necrotizing pancreatitis was present in 31 patients (34.1\%). Twenty-seven (29.7\%) patients developed systemic inflammatory response syndrome and 42
(46.2\%) patients presented organ failure, the most common being due to respiratory failure $(n=30 ; 33.0 \%)$ followed by renal failure $(n=10 ; 11.0 \%)$.

\section{Determinants of severity in acute pancreatitis}

Both groups of patients with severe and mild AP were comparable in terms of age $(64.8 \pm 16.3$ years vs $67.9 \pm$ 13.7 years; $p=0.239$ ) and gender (males: $58.2 \%$ vs $51.6 \%$; $p=0.228$ ), as described in Table 1 . After multivariate analysis of significant risk factors in univariate analysis, no significant differences were verified in relation to hospital stay or comorbidities between two groups. Concerning laboratory parameters, there was a significant association of severe AP with high BUN (26.6 \pm 17.1 vs $19.4 \pm 8.8$ years;aOR $=1.002 ; p<0.004)$, serum glucose $\quad(168.0 \pm 72.6 \quad$ vs $130.9 \pm 35.5 ; \mathrm{aOR}=1.002$; $p<0.001), \mathrm{RDW}_{\mathrm{Oh}}(14.6 \pm 1.3$ vs $12.7 \pm 0.5$; $\mathrm{aOR}=1.129$; $p<0.001), \mathrm{RDW}_{24 \mathrm{~h}}(14.3 \pm 1.9$ vs $12.8 \pm 0.5$; aOR $=1.015$; $p=0.005)$ and $\mathrm{RDW}_{0 \mathrm{~h}}$-to-TSC ratio $(1.8 \pm 0.4$ vs $1.3 \pm$ 0.1 ; $\mathrm{aOR}=1.556 ; p<0.001)$. In addition, there was also a significant association of severe AP with conventional prognostic scores, namely Ranson $(2.6 \pm 1.2$ years vs 1.5 $\pm 0.9 ; \mathrm{aOR}=1.043 ; p<0.001)$, BISAP $(1.7 \pm 0.9$ vs $1.0 \pm$ $0.7 ; \mathrm{aOR}=1.028 ; p<0.001)$ and $\mathrm{MM}(0.8 \pm 0.7$ vs $0.0 \pm$ $0.0 ; \mathrm{aOR}=1.184 ; p<0.001)$ scores (Table 1$)$.

\section{Determinants of mortality in acute pancreatitis}

The overall mortality rate was $8.8 \%(16 / 182)$, all cases associated with severe AP, corresponding to a mortality rate of $17.6 \%(16 / 91)$ in the severe AP group. As described in Table 2, cases and controls were similar and therefore comparable in terms of age $(71.6 \pm 14.3$ years vs $65.8 \pm 15.1$ years; $p=0.123)$ and gender $(68.8 \%$ vs $53.6 \% ; p=0.245)$. After multivariate analysis, there were 
Table 1 Characterization of population according to acute pancreatitis severity

\begin{tabular}{|c|c|c|c|c|}
\hline Variable & Severe acute pancreatitis $(n=91)$ & Mild acute pancreatitis $(n=91)$ & $p U / p M$ & $\mathrm{aOR}, 95 \mathrm{Cl}$ in $\mathrm{M}$ \\
\hline Age (yo), $\mu \pm \sigma$ & $64.8 \pm 16.3$ & $67.9 \pm 13.7$ & $0.239 /-$ & - \\
\hline Male gender, n(\%) & $53(58.2 \%)$ & $47(51.6 \%)$ & $0.228 /-$ & - \\
\hline Alcoholism, n(\%) & $26(28.6 \%)$ & $14(15.4 \%)$ & $0.032 /-$ & - \\
\hline Smoking, n(\%) & $0(0.0 \%)$ & $5(5.5 \%)$ & $0.023 /-$ & - \\
\hline Hospital stay, median(IQR) & $1.3(2-83)$ & $8.0(2-25)$ & $0.001 /-$ & - \\
\hline Comorbidities ${ }^{a}, \mathrm{n}(\%)$ & $75(82.4 \%)$ & $83(91.2 \%)$ & $0.08 /-$ & - \\
\hline Hypothyroidism & $2(2.2 \%)$ & $9(9.9 \%)$ & $0.029 /-$ & - \\
\hline Cerebral vascular accident & $9(9.9 \%)$ & $0(0.0 \%)$ & $0.002 /-$ & - \\
\hline Chronic renal disease & $10(11.0 \%)$ & $3(3.3 \%)$ & $0.004 /-$ & - \\
\hline \multicolumn{5}{|l|}{ Laboratory and gasometrical parameters } \\
\hline WBC count (cells/mm³), $\mu \pm \sigma$ & $15,630.0 \pm 9030.0$ & $31,165.0 \pm 1631.7$ & $0.001 /-$ & - \\
\hline AST (IU/L), $\mu \pm \sigma$ & $193.5 \pm 164.1$ & $778.9 \pm 354.6$ & $0.003 /-$ & - \\
\hline $\operatorname{ALT}(I U / L), \mu \pm \sigma$ & $249.7 \pm 180.3$ & $445.5 \pm 252.8$ & $0.129 /-$ & - \\
\hline Total bilirubin (mg/dL), $\mu \pm \sigma$ & $2.7 \pm 2.6$ & $2.2 \pm 1.9$ & $0.363 /-$ & - \\
\hline Platelet count (cells $\left./ \mathrm{mm}^{3}\right), \mu \pm \sigma$ & $212.2 \pm 87.1$ & $208.8 \pm 72.8$ & $0.975 /-$ & - \\
\hline $\mathrm{BUN}(\mathrm{mg} / \mathrm{dL}), \mu \pm \sigma$ & $26.6 \pm 17.1$ & $19.4 \pm 8.8$ & $0.009 /<0.001$ & $1.002,[1.001 ; 1.007]$ \\
\hline Creatinine $(\mathrm{mg} / \mathrm{dL}), \mu \pm \sigma$ & $1.5 \pm 1.3$ & $0.9 \pm 0.3$ & $<0.001 /-$ & - \\
\hline INR, $\mu \pm \sigma$ & $3.0 \pm 1.6$ & $1.4 \pm 0.2$ & $<0.001 /-$ & - \\
\hline Albumin (g/dL), $\mu \pm \sigma$ & $3.6 \pm 0.6$ & $4.0 \pm 0.7$ & $<0.001 /-$ & - \\
\hline $\mathrm{LDH}(\mathrm{IU} / \mathrm{L}), \mu \pm \sigma$ & $503.0 \pm 403.7$ & $406.8 \pm 305.4$ & $<0.001 /-$ & - \\
\hline Serum glucose (mg/dL), $\mu \pm \sigma$ & $168.0 \pm 72.6$ & $130.9 \pm 35.5$ & $<0.001 /<0.001$ & $1.002,[1.001 ; 1.317]$ \\
\hline Hemoglobin (mg/dL), $\mu \pm \sigma$ & $13.8 \pm 2.6$ & $13.5 \pm 1.4$ & $0.406 /-$ & - \\
\hline Hematocrit (\%), $\mu \pm \sigma$ & $41.5 \pm 7.0$ & $40.2 \pm 4.3$ & $0.285 /-$ & - \\
\hline Serum amylase (IU/L), $\mu \pm \sigma$ & $1315.2 \pm 1163.3$ & $2049.2 \pm 1715.0$ & $0.398 /-$ & - \\
\hline $\mathrm{CRP}_{\mathrm{oh}}(\mathrm{mg} / \mathrm{dL}), \mu \pm \sigma$ & $10.9 \pm 9.2$ & $5.0 \pm 3.3$ & $<0.001 /-$ & - \\
\hline $\mathrm{CRP}_{24 \mathrm{~h}}(\mathrm{mg} / \mathrm{dL}), \mu \pm \sigma$ & $21.1 \pm 11.6$ & $11.3 \pm 5.8$ & $<0.001 /-$ & - \\
\hline Lactate $(\mathrm{mg} / \mathrm{dL}), \mu \pm \sigma$ & $2.8 \pm 1.7$ & $1.5 \pm 1.0$ & $<0.001 /-$ & - \\
\hline D-dimers $(\mu \mathrm{g} / \mathrm{mL}), \mu \pm \sigma$ & $6.6 \pm 6.5$ & $1.5 \pm 0.3$ & $0.192 /-$ & - \\
\hline Total serum calcium (mg/dL), $\mu \pm \sigma$ & $8.2 \pm 1.2$ & $9.6 \pm 0.4$ & $<0.001 /-$ & - \\
\hline RDW $_{\text {oh }}(\%), \mu \pm \sigma$ & $14.6 \pm 1.3$ & $12.7 \pm 0.5$ & $<0.001 /<0.001$ & $1.129,[1.065 ; 1.192]$ \\
\hline $\mathrm{RDW}_{24 \mathrm{~h}}(\%), \mu \pm \sigma$ & $14.3 \pm 1.9$ & $12.8 \pm 0.5$ & $<0.001 / 0.005$ & $1.015,[1.003 ; 1.055]$ \\
\hline $\mathrm{RDW}_{\text {oh }}$-to-total serum calcium ratio, $\mu \pm \sigma$ & $1.8 \pm 0.4$ & $1.3 \pm 0.1$ & $<0.001 /<0.001$ & $1.556,[1.356 ; 1.756]$ \\
\hline $\mathrm{RDW}_{\text {oh}}$-to-platelets ratio, $\mu \pm \sigma$ & $0.08 \pm 0.03$ & $0.07 \pm 0.02$ & $0.03 /-$ & - \\
\hline \multicolumn{5}{|l|}{ Prognostic scores } \\
\hline Ranson score, $\mu \pm \sigma$ & $2.6 \pm 1.2$ & $1.5 \pm 0.9$ & $<0.001 /<0.001$ & $1.043,[1.001 ; 1.088]$ \\
\hline BISAP score, $\mu \pm \sigma$ & $1.7 \pm 0.9$ & $1.0 \pm 0.7$ & $<0.001 /<0.001$ & $1.028,[1.016 ; 1.092]$ \\
\hline Modified Marshall score, $\mu \pm \sigma$ & $0.8 \pm 0.7$ & $0.0 \pm 0.0$ & $<0.001 /<0.001$ & $1.184,[1.112 ; 1.256]$ \\
\hline
\end{tabular}

$A L T$ alanine aminotransferase, $A S T$ aspartate aminotransferase, $a O R$ adjusted odds ratio, $B U N$ blood urea nitrogen, $C R P_{o h} \mathrm{C}$-reactive protein at $0 \mathrm{~h}, C R P_{24 h} \mathrm{C}$-reactive protein at $24 \mathrm{~h}, \mathrm{Cl}$ confidence interval, INR international normalized ratio, IQR interquartile range, $L D H$ lactate dehydrogenase, $M$ multivariate analysis, $R D W_{O h}$ red cell distribution width at $0 \mathrm{~h}, R D W_{24 h}$ red cell distribution width at $24 \mathrm{~h}, \mathrm{U}$ univariate analysis, $W B C$ white blood cells

a Only presented significant comorbidities in univariate analysis; Comorbidities evaluated: Neurological, cardiovascular, respiratory and gastrointestinal conditions, malignancy and immunosuppression

no significant differences between survivors and nonsurvivors regarding hospital stay and comorbidities. Nonetheless, nonsurvivors had higher lactate at admission
$(3.6 \pm 1.8$ vs $2.0 \pm 1.4 ; \mathrm{aOR}=1.164 ; p=0.019), \quad \mathrm{RDW}_{0 \mathrm{~h}}$ $(15.3 \pm 1.4$ vs $13.5 \pm 1.3 ; \mathrm{aOR}=1.038 ; p<0.001), \mathrm{RDW}_{24 \mathrm{~h}}$ $(15.1 \pm 1.4 \quad$ vs $13.4 \pm 1.6 ; \mathrm{aOR}=1.006 ; p=0.005) \quad$ and 
Table 2 Characterization of population according to acute pancreatitis mortality

\begin{tabular}{|c|c|c|c|c|}
\hline Variable & Nonsurvivors $(n=16)$ & Survivors $(n=166)$ & $p \cup / p M$ & $\mathrm{aOR}, 95 \% \mathrm{Cl}$ in $\mathrm{M}$ \\
\hline Age (yo), $\mu \pm \sigma$ & $71.6 \pm 14.3$ & $65.8 \pm 15.1$ & $0.123 /-$ & - \\
\hline Male gender, n(\%) & $11(68.8 \%)$ & $5(53.6 \%)$ & $0.245 /-$ & - \\
\hline Alcoholism, n(\%) & $4(25.0 \%)$ & $36(21.7 \%)$ & $0.760 /-$ & - \\
\hline Smoking, n(\%) & $0(0.0 \%)$ & 16(9.6\%) & $0.481 /-$ & - \\
\hline Hospital stay, median(IQR) & $6.5(2-62)$ & $11.0(2-83)$ & $0.071 /-$ & - \\
\hline Comorbidities $^{a}, \mathrm{n}(\%)$ & 15(93.7\%) & 143(86.1\%) & $0.391 /-$ & - \\
\hline \multicolumn{5}{|l|}{ Laboratory and gasometrical parameters } \\
\hline WBC count (cells/mm³), $\mu \pm \sigma$ & $16,810.0 \pm 13,490.0$ & $1779.1 \pm 1214.1$ & $0.465 /-$ & - \\
\hline AST (IU/L), $\mu \pm \sigma$ & $144.6 \pm 132.4$ & $271.6 \pm 198.1$ & $0.416 /-$ & - \\
\hline $\operatorname{ALT}(I \cup / L), \mu \pm \sigma$ & $123.2 \pm 100.2$ & $227.8 \pm 175.4$ & $0.116 /-$ & - \\
\hline Total bilirubin (mg/dL), $\mu \pm \sigma$ & $1.8 \pm 1.2$ & $2.4 \pm 2.3$ & $0.869 /-$ & - \\
\hline Platelet count (cells $/ \mathrm{mm}^{3}$ ), $\mu \pm \sigma$ & $174.4 \pm 67.0$ & $214.0 \pm 80.5$ & $0.057 /-$ & - \\
\hline $\mathrm{BUN}(\mathrm{mg} / \mathrm{dL}), \mu \pm \sigma$ & $35.3 \pm 20.1$ & $21.9 \pm 12.5$ & $0.002 /-$ & - \\
\hline Creatinine (mg/dL), $\mu \pm \sigma$ & $2.2 \pm 1.4$ & $1.1 \pm 0.8$ & $<0.001 /-$ & - \\
\hline INR, $\mu \pm \sigma$ & $1.5 \pm 1.0$ & $2.1 \pm 1.2$ & $0.018 /-$ & - \\
\hline Albumin ( $\mathrm{g} / \mathrm{dL}), \mu \pm \sigma$ & $3.5 \pm 0.6$ & $3.8 \pm 0.7$ & $0.074 /-$ & - \\
\hline $\mathrm{LDH}(\mathrm{IU} / \mathrm{L}), \mu \pm \sigma$ & $465.1 \pm 287.8$ & $453.9 \pm 332.7$ & $0.207 /-$ & - \\
\hline Serum glucose $(\mathrm{mg} / \mathrm{dL}), \mu \pm \sigma$ & $164.1 \pm 104.5$ & $147.3 \pm 53.2$ & $0.945 /-$ & - \\
\hline Hemoglobin (mg/dL), $\mu \pm \sigma$ & $13.7 \pm 3.0$ & $13.7 \pm 2.0$ & $0.889 /-$ & - \\
\hline Hematocrit (\%), $\mu \pm \sigma$ & $41.7 \pm 7.5$ & $40.7 \pm 5.7$ & $0.630 /-$ & - \\
\hline Serum amylase (IU/L), $\mu \pm \sigma$ & $1565.1 \pm 1273.3$ & $1703.4 \pm 1506.4$ & $0.391 /-$ & - \\
\hline $\mathrm{CRP}_{\mathrm{Oh}}(\mathrm{mg} / \mathrm{dL}), \mu \pm \sigma$ & $10.4 \pm 9.6$ & $5.9 \pm 3.9$ & $0.046 /-$ & - \\
\hline $\mathrm{CRP}_{24 \mathrm{~h}}(\mathrm{mg} / \mathrm{dL}), \mu \pm \sigma$ & $21.8 \pm 10.4$ & $15.7 \pm 10.3$ & $0.028 /-$ & - \\
\hline Lactate $(\mathrm{mg} / \mathrm{dL}), \mu \pm \sigma$ & $3.6 \pm 1.8$ & $2.0 \pm 1.4$ & $<0.001 / 0.019$ & $1.164,[1.003 ; 1.474]$ \\
\hline D-dimers $(\mu \mathrm{g} / \mathrm{mL}), \mu \pm \sigma$ & $9.1 \pm 6.8$ & $5.9 \pm 4.6$ & $0.042 /-$ & - \\
\hline Total serum calcium (mg/dL), $\mu \pm \sigma$ & $8.0 \pm 1.1$ & $8.9 \pm 1.1$ & $0.001 /-$ & - \\
\hline $\mathrm{RDW}_{\text {oh }}(\%), \mu \pm \sigma$ & $15.3 \pm 1.4$ & $13.5 \pm 1.3$ & $<0.001 /<0.001$ & $1.038,[1.011 ; 1.087]$ \\
\hline $\mathrm{RDW}_{24 h}(\%), \mu \pm \sigma$ & $15.1 \pm 1.4$ & $13.4 \pm 1.6$ & $<0.001 / 0.005$ & 1.006, [1.001;1.040] \\
\hline $\mathrm{RDW}_{\text {oh}}$-to-total serum calcium ratio, $\mu \pm \sigma$ & $2.0 \pm 0.3$ & $1.6 \pm 0.3$ & $<0.001 /<0.001$ & $1.018,[1.007 ; 1.135]$ \\
\hline $\mathrm{RDW}_{\text {oh-to-platelets ratio, }} \mu \pm \sigma$ & $0.10 \pm 0.04$ & $0.07 \pm 0.03$ & $0.015 /-$ & \\
\hline \multicolumn{5}{|l|}{ Prognostic scores } \\
\hline Ranson score, $\mu \pm \sigma$ & $2.7 \pm 1.2$ & $2.0 \pm 1.2$ & $0.023 /<0.001$ & $1.019,[1.005 ; 1.170]$ \\
\hline BISAP score, $\mu \pm \sigma$ & $2.0 \pm 1.0$ & $1.3 \pm 0.8$ & $0.003 /<0.001$ & $1.010,[1.003 ; 1.059]$ \\
\hline Modified Marshall score, $\mu \pm \sigma$ & $1.2 \pm 1.0$ & $0.6 \pm 0.3$ & $<0.001 / 0.020$ & 1.109, [1.049;1.169] \\
\hline
\end{tabular}

$A L T$ alanine aminotransferase, $A S T$ aspartate aminotransferase, $a O R$ adjusted odds ratio, $B U N$ blood urea nitrogen, $C R P_{0 h} \mathrm{C}$-reactive protein at $0 \mathrm{~h}, C R P_{24 h} \mathrm{C}$-reactive protein at $24 \mathrm{~h}, \mathrm{Cl}$ confidence interval, INR international normalized ratio, IQR interquartile range, $L D H$ lactate dehydrogenase, $M$ multivariate analysis, $R D W_{\text {oh }}$ red cell distribution width at $0 \mathrm{~h}, R D W_{24 h}$ red cell distribution width at $24 \mathrm{~h}, U$ univariate analysis, WBC white blood cells

a Only presented significant comorbidities in univariate analysis; Comorbidities evaluated: Neurological, cardiovascular, respiratory and gastrointestinal conditions, malignancy and immunosuppression

$\mathrm{RDW}_{\mathrm{Oh}}$-to-TSC ratio $(2.0 \pm 0.3$ vs $1.6 \pm 0.3 ; \mathrm{aOR}=1.018 ; p$ $<0.001)$ than survivors. Concerning usual prognostic scores, Ranson $(2.7 \pm 1.2$ vs $2.0 \pm 1.2$; aOR $=1.019 ; p<$ $0.001)$, BISAP $(2.0 \pm 1.0$ vs $1.3 \pm 0.8 ; \mathrm{aOR}=1.010 ; p<$ $0.001)$ and $\mathrm{MM}(1.2 \pm 1.0$ vs $0.6 \pm 0.3 ; \mathrm{aOR}=1.109 ; p=$ $0.020)$ scores were also significantly higher in nonsurvivors.

\section{Discriminating performance of RDW and other validated prognostic scores for severity and mortality in acute pancreatitis}

In order to assess the discriminant performance of RDW in terms of severity and mortality in AP and to establish a comparison with other independent risk factors, an AUROC analysis was performed. RDW $_{\text {oh }}$ (AUROC:0.960; $p$ 
$<0.001$ ) and $\mathrm{RDW}_{0 \mathrm{~h}}$-to-TSC ratio (AUROC:0.973; $p<$ 0.001 ) were major predictors of severe AP to a cut-off value of 13.0 (S-92.7\%; Sp-84.3\%) and 1.4 (S-96.3\%; Sp-84.3\%), respectively. These factors were superior to well-stablished prognostic scores as Ranson (AUROC: 0.777; $p<0.001$; cut-off:3.0), MM (AUROC: 0.756; $p<0.001$; cut-off:1.0) and BISAP (AUROC: 0.732; $p<0.001$; cut-off:2.0) (Fig. 2).

Relatively to mortality in AP and as verified for AP severity, RDW at $0 \mathrm{~h}$ and $24 \mathrm{~h}$ post-admission were the best predictors for mortality in AP (AUROC: 0.842; $p<0.001$; cut-off: 14.0 and AUROC: $0.848 ; p<0.001$; cutoff:13.8, respectively), followed by $\mathrm{RDW}_{0 \mathrm{~h}}$-to-TSC ratio (AUROC: $0.820 ; p<0.001$; cut-off: 1.7 ). All of these parameters were better predictors of AP mortality than MM (AUROC: $0.806 ; p<0.001$; cut-off:1.0), BISAP (AUROC: 0.693; $p=0.017$; cut-off:2.0) or Ranson (AUROC: 0.640; $p=0.003$; cut-off: 3.0 ) scores (Fig. 3).

\section{Discussion}

Our study expands the knowledge about early noninvasive predictors of severity and mortality in AP, namely RDW and RDW-to-TSC ratio, since they are simple, inexpensive, quantitative and easy to determine. Furthermore, they proved to have a good-excellent accuracy better than specific-AP prognostic scores, such as
Ranson, BISAP and MM, after excluding conditions susceptible to influence RDW and adjusting for confounders.

The assessment of AP severity is crucial to improve prognosis $[2,4,17]$. However, $20-30 \%$ of severe AP are misdiagnosed based on clinical data, imaging and biochemical analysis [18]. Over the past decades, several scoring systems have been proposed for early patients' stratification who have an increased risk of AP morbidity and mortality, with similar accuracy between them [5]. However, no single prognostic score is available for early assessment of AP severity in clinical practice [11]. Two of major scores studied and widely used were Ranson [19] and BISAP [20], since they can be determined in the first $24 \mathrm{~h}$ (although Ranson score is only completed within $48 \mathrm{~h}$ ) and they were specifically developed for AP comparing to others, such as Acute Physiology and Chronic Health Evaluation (APACHE-II) score, which was developed to manage critically ill patients in intensive care units. Accuracy reported for Ranson and BISAP scores were 0.69 and 0.74 , respectively [5]. MM score was also recently used to determine AP severity [3]. Other single serum markers of AP severity have been extensively studied, such as hematocrit, creatinine, BUN, TSC, lactate and CRP $[4,7,21-23]$. In addition, some serum pro- and anti-inflammatory markers have been

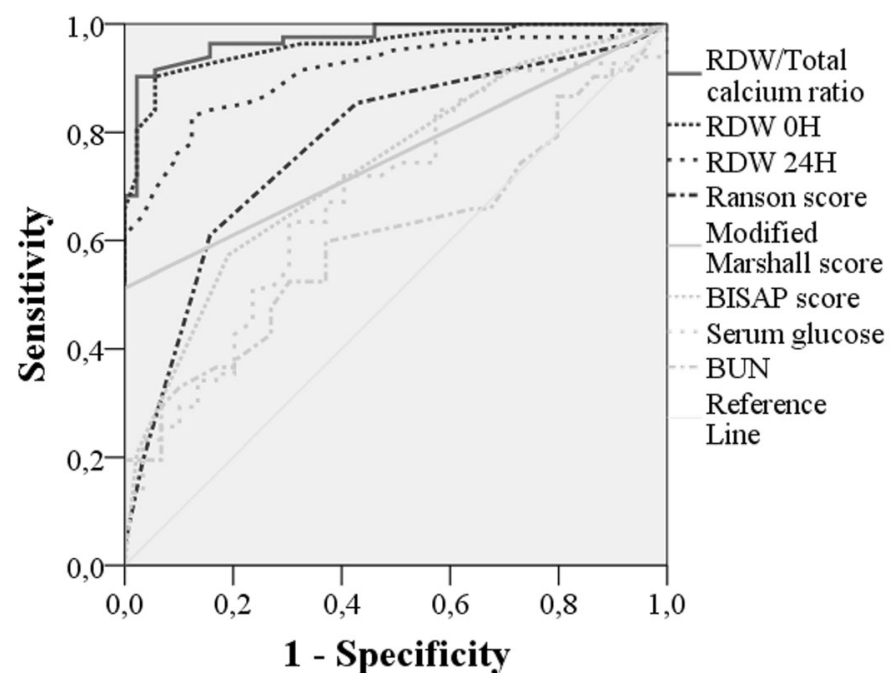

\begin{tabular}{|r|c|c|c|c|c|c|}
\hline & AUROC & $\mathrm{p}$ & $95 \%$ CI & cut-off & Sensitivity & Specificity \\
\hline $\begin{array}{r}\mathrm{RDW}_{\text {oh }} \text {-to-total serum } \\
\text { calcium ratio }\end{array}$ & 0.973 & $<0.001$ & {$[0.952 ; 0.994]$} & 1.4000 & $96.3 \%$ & $84.3 \%$ \\
\hline $\mathrm{RDW}_{0 \mathrm{~h}}$ & 0.960 & $<0.001$ & {$[0.932 ; 0.988]$} & 13.050 & $92.7 \%$ & $84.3 \%$ \\
\hline $\mathrm{RDW}_{24 \mathrm{~h}}$ & 0.910 & $<0.001$ & {$[0.864 ; 0.955]$} & 12.950 & $86.6 \%$ & $74.2 \%$ \\
\hline MANSON score & 0.777 & $<0.001$ & {$[0.705 ; 0.848]$} & 1.500 & $71.4 \%$ & $64.8 \%$ \\
\hline Modified Marshal score & 0.756 & $<0.001$ & {$[0.680 ; 0.832]$} & 1.000 & $51.2 \%$ & $100.0 \%$ \\
\hline BISAP score & 0.732 & $<0.001$ & {$[0.657 ; 0.807]$} & 1.500 & $57.3 \%$ & $55.1 \%$ \\
\hline Serum glucose & 0.681 & $<0.001$ & {$[0.600 ; 0.761]$} & 127.000 & $72.0 \%$ & $59.6 \%$ \\
\hline BUN & 0.606 & 0.016 & {$[0.520 ; 0.693]$} & 19.500 & $59.8 \%$ & $37.1 \%$ \\
\hline RDW, red cell distribution width; BUN, blood urea nitrogen; CI, confidence interval \\
\hline
\end{tabular}

Fig. 2 Area under the receiver operating characteristic curve (AUROC) of prognostic scores and independent risk factors for acute pancreatitis severity 


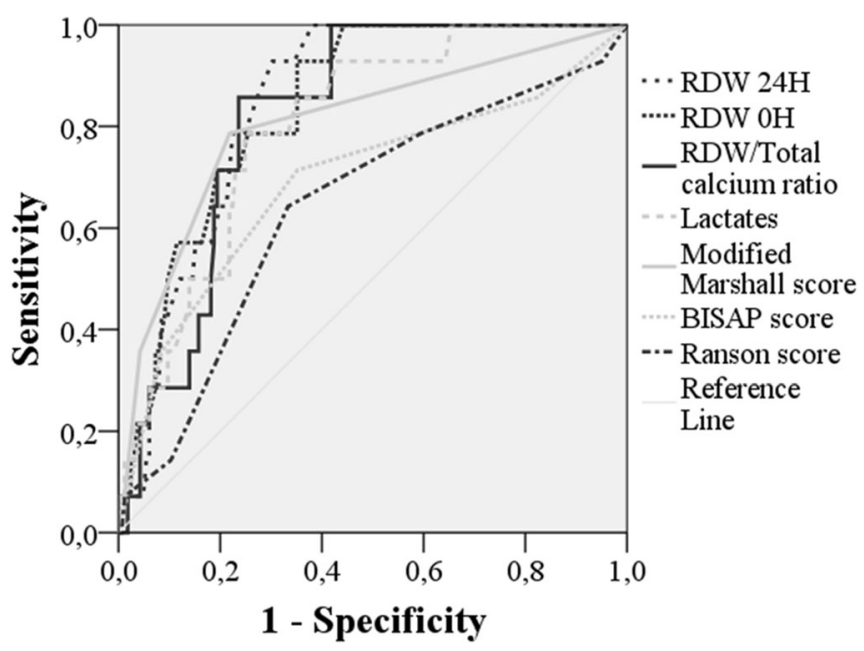

\begin{tabular}{|r|c|c|c|c|c|c|}
\hline & AUROC & $\mathrm{p}$ & $95 \% \mathrm{CI}$ & cut-off & Sensitivity & Specificity \\
\hline $\mathrm{RDW}_{24 \mathrm{~h}}$ & 0.848 & $<0.001$ & {$[0.780 ; 0.916]$} & 13.850 & $85.7 \%$ & $72.7 \%$ \\
\hline $\mathrm{RDW}_{\text {oh }}$ & 0.842 & $<0.001$ & {$[0.762 ; 0.921]$} & 14.050 & $78.6 \%$ & $74.5 \%$ \\
\hline $\begin{array}{r}\mathrm{RDW}_{\text {oh }} \text {-to-total serum } \\
\text { calcium ratio }\end{array}$ & 0.820 & $<0.001$ & {$[0.743 ; 0.897]$} & 1.671 & $85.7 \%$ & $76.4 \%$ \\
\hline Lactate & 0.801 & $<0.001$ & {$[0.702 ; 0.900]$} & 2.370 & $78.6 \%$ & $75.2 \%$ \\
\hline Modified Marshal score & 0.806 & $<0.001$ & {$[0.672 ; 0.939]$} & 1.000 & $78.6 \%$ & $78.2 \%$ \\
\hline BISAP score & 0.693 & 0.017 & {$[0.526 ; 0.859]$} & 1.500 & $71.4 \%$ & $64.8 \%$ \\
\hline RANSON score & 0.640 & 0.003 & {$[0.483 ; 0.797]$} & 2.500 & $64.3 \%$ & $66.7 \%$ \\
\hline RDW, red cell distribution width; CI, confidence interval \\
\hline
\end{tabular}

Fig. 3 Area under the receiver operating characteristic curve (AUROC) of prognostic scores and independent risk factors for acute pancreatitis mortality

proved to be associated with AP severity, such as interleukin-6, interlekin-8, interleukin-10 and tumor necrosis factor alpha; and serum procalcitonin, a reliable marker of infection/sepsis and useful for predicting infected necrosis in AP. However, the main handicaps of these markers are the fact that they are not routinely used and associated with significant additional costs $[2,6,22]$.

The association between arterial lactate and AP mortality was poorly studied [23]. Our study showed that lactate $>2.4 \mathrm{mg} / \mathrm{dL}$ (AUROC 0.801 ) was strongly associated with AP mortality, similar to other work with a reported AUROC of 0.87 [23].

RDW has been considered a remarkable prognostic marker to determine mortality risk in several conditions beyond AP, reflecting inflammation status [8-11]. In a recent systematic review, RDW was independently associated with AP mortality [18]. However, none of published works compared RDW with common prognostic scores [12]. The present study showed that RDW at admission and in the first $24 \mathrm{~h}$ were good predictors of AP mortality with AUROC $>0.842$, which was higher than in other works with a reported AUROC of 0.66-0.82 [10-12]. For the first time, we also found a strong and independent correlation between $\mathrm{RDW}_{\mathrm{Oh}}$-to-TSC ratio and AP mortality with AUROC of 0.820. In our study, RDW-to-TSC ratio and RDW at admission were superior to conventional prognostic scores in determining AP mortality. Çetinkaya et al. also studied the influence of RDW-to-platelet ratio on AP mortality with an AUROC of 0.783 to a cut-off value of 0.00067 [24]. This marker was also studied in our work, but despite the significance in univariate analysis, it was not an independent prognostic factor after multivariate analysis.

As described in the literature, the present study showed that all AP-associated deaths occurred in patients with severe AP $[1,4]$, suggesting that finding predictors of AP severity is also crucial in addition to predicting mortality in AP. Despite BUN and serum glucose were associated with AP severity in our study, they did not show a good predictive power (AUROC 0.640 and 0.693 , respectively). This association was also verified in other work, with sensitivity of $79 \%$ for BUN and $67 \%$ for serum glucose [25]. In a recent work, other biochemical parameters were associated with persistent organ failure in AP, including serum albumin with an AUROC slightly higher than Ranson score (0.873 and 0.845 , respectively) [26]. However, serum albumin did not reach statistical significance in our study. Regarding RDW, only two studies assessed the relation between this factor and AP severity [14, 15]. A recent work found that RDW could be a useful indicator of AP severity better than serum glucose or TSC (AUROC of 0.801, 0.658 and 0.227 , respectively) [15]. However, another recent 
study concluded that RDW was not a predictor for AP severity, contrarily to BISAP or TSC [14]. In this work, an excellent predictability of AP severity by $\mathrm{RDW}_{0 \mathrm{~h}}$ (AUROC 0.960) was verified. For the first time, we also showed a strong and positive association between $\mathrm{RDW}_{0 \mathrm{~h}}$-to-TSC ratio and AP severity, representing the best predictor of it (AUROC 0.973). In addition, we verified that conventional AP-specific prognostic scores were reasonable predictors for AP severity (AUROC of 0.777, 0.756 and 0.732 for Ranson, MM and BISAP scores, respectively).

In the past, RDW was widely used for differential diagnosis of anemia. Over the last years, RDW has been associated with systemic inflammation [27] due to the high oxidative stress and inflammatory cytokines that contribute to RDW elevation by reducing RBC survival and maturation, increasing the release of newer and larger $\mathrm{RBC}$ into the peripheral circulation and changing of membrane glycoproteins and ion channels of RBC with consequent morphological alteration $[10,12,28]$. Therefore, RDW reflects the degree of inflammation that occurs in AP and thus, can be used to predict its severity. Despite isolated TSC did not represent a good predictor for AP severity in our study, $\mathrm{RDW}_{0 \mathrm{~h}}$-to-TSC ratio proved to be an excellent predictor of AP severity and a very good predictor of AP mortality. In fact, hypocalcemia within the first $24 \mathrm{~h}$ was associated with AP severity, although its etiopathogenesis is not clearly understood. It has been postulated that hypocalcemia could be related to the calcium soaps formation and parathyroid hormone depletion [21].

There are some limitations, mainly related to the retrospective nature of this study. However, our study groups' size is within range when compared to other published studies for assessing both severity or mortality in AP $[10-12,14,15]$. Second, RDW samples were collected from a single center and thus, RDW levels could have been slightly different in other populations studied.

\section{Conclusions}

RDW and TSC are simple, inexpensive, noninvasive and quantitative serum markers, provided in a complete blood count test, and therefore readily available on admission. Our study highlights the good predictive power of RDW, evaluated at admission and in the first $24 \mathrm{~h}$, as well as $\mathrm{RDW}_{\mathrm{Oh}}$-to-TSC ratio for both severity and mortality in AP, being superior to Ranson, BISAP and MM scores. $\mathrm{RDW}_{0 \mathrm{O}}>$ 13.0 and RDW -to-TSC $>1.4$ were excellent predictors for AP severity. $\mathrm{RDW}_{0 \mathrm{~h}}>14.0$ and RD0h-to-TSC $>1.7$ were very-good predictors for AP mortality.

Further prospective and multicenter studies are needed to more accurately assess the impact of high RDW as a predictor of severity and mortality in AP and to understand the pathophysiological mechanisms underlying RDW and AP prognosis.

\section{Abbreviations}

ABG: Arterial blood gas; ALT: Alanine aminotransferase; aOR: Adjusted odds ratio; AP: Acute pancreatitis; AST: Aspartate aminotransferase; AUROC: Area under the receiver operating characteristic curve; BISAP: Bedside Index for Severity in Acute Pancreatitis; BUN: Blood urea nitrogen; CRP: C-reactive protein; $\mathrm{CRP}_{0 \mathrm{~h}}$ : C-reactive protein at $0 \mathrm{~h} ; \mathrm{CRP}_{24 \mathrm{~h}}$ : C-reactive protein at $24 \mathrm{~h}$; INR: International normalized ratio; LDH: Lactate dehydrogenase;

MM: Modified Marshall score system; RAC: The revised Atlanta classification 2012; RBC: Red blood cell; RDW: Red cell distribution width; RDWoh: Red cell distribution width at $0 \mathrm{~h} ; \mathrm{RDW}_{24 \mathrm{~h}}$ : Red cell distribution width at $24 \mathrm{~h}$;

SD: Standard deviation; TSC: Total serum calcium; WBC: White blood cells

\section{Availability of data and materials}

To ensure that data confidentiality is not compromised, the dataset supporting the results of this article will not be integrated in the manuscript. The datasets are available from the corresponding author on reasonable request.

\section{Guarantor of the article}

Marta Gravito-Soares.

\section{Authors' contributions}

The involvement of each author was as follows: MGS (1-8); EGS (1-8); DG $(1,5,6,7,8) ;$ NA $(1,3,5,7,8) ;$ LT (5,8). Key: (1) Study concept and design; (2) Acquisition of data; (3) Analysis and interpretation of data; (4) Drafting of the manuscript; (5) Critical revision of the manuscript for important intellectual content; (6) Statistical analysis; (7) Administrative, technical, or material support; (8) Study supervision. All authors read the final version of this article, approve its' content and submission for publication. All authors agreed to be accountable for all aspects of the work in ensuring that questions related to the accuracy or integrity of any part of the work are appropriately investigated and resolved.

\section{Ethics approval and consent to participate}

The authors declare that no experiments were performed on humans or animals for this study. The study was approved by the Ethics Committee of Centro Hospitalar e Universitário de Coimbra and was performed in accordance with the Declaration of Helsinki. They have followed the protocols of their work center on the publication of patient data. Patient consent was not obtained since all data was retrieved retrospectively from medical records without additional blood samples or biochemical analysis.

\section{Consent for publication}

Not applicable. The authors declare that no patient data (details, images or videos relating to individual participants) are included in this article.

\section{Competing interests}

The authors declare that they have no competing interests.

\section{Publisher's Note}

Springer Nature remains neutral with regard to jurisdictional claims in published maps and institutional affiliations.

Received: 6 October 2017 Accepted: 26 June 2018

Published online: 05 July 2018

\section{References}

1. Yadav D, Lowenfels AB. The epidemiology of pancreatitis and pancreatic cancer. Gastroenterol. 2013;144:1252-61.

2. Wang X, Cui Z, Zhang J, Li H, Zhang D, Miao B, et al. Early predictive factors of in hospital mortality in patients with severe acute pancreatitis. Pancreas. 2010;39:114-5.

3. Banks PA, Bollen TL, Dervenis C, Gooszen HG, Johnson CD, Sarr MG, et al. Classification of acute pancreatitis-2012: revision of the Atlanta classification and definitions by international consensus. Gut. 2013;62:102-11.

4. Almeida N, Fernandes A, Casela A. Predictors of severity and in-hospital mortality for acute pancreatitis: is there any role for C-reactive protein determination in the first 24 hours? GE Port J Gastroenterol. 2015;22:187-9.

5. Cho JH, Kim TN, Chung HH, Kim KH. Comparison of scoring systems in predicting the severity of acute pancreatitis. World J Gastroenterol. 2015;21: 2387-94. 
6. Pavlidis TE, Pavlidis EJ, Sakantamis AK. Advances in prognostic factors in acute pancreatitis: a mini-review. Hepatobiliany Pancreat Dis Int. 2010;9:482-6.

7. Cardoso FS, Ricardo LB, Oliveira AM, Canena JM, Horta DV, Papoila AL, et al. C-reactive protein prognostic accuracy in acute pancreatitis: timing of measurement and cutoff points. Eur J Gastroenterol Hepatol. 2013;25:784-9.

8. Patel KV, Semba RD, Ferrucci L, Newman AB, Fried LP, Wallace RB, et al. Red cell distribution width and mortality in older adults: a meta-analysis. J Gerontol A Biol Sci Med Sci. 2010;65:258-65.

9. Hu ZD, Chen Y, Zhang L, Sun Y, Huang YL, Wang QQ, et al. Red blood cell distribution width is a potential index to assess the disease activity of systemic lupus erythematous. Clin Chim Acta. 2013;425:202-5.

10. Senol K, Saylam B, Kocaay F, Tez M. Red cell distribution width as a predictor of mortality in acute pancreatitis. Am J Emerg Med. 2013;31:687-9.

11. Wang D, Yang J, Zhang J, Zhang S, Wang B, Wang R, et al. Red cell distribution width predicts deaths in patients with acute pancreatitis. J Res Med Sci. 2015;20:424-8.

12. Goyal H, Awad H, Hu ZD. Prognostic value of admission red blood cell distribution width in acute pancreatitis: a systematic review. Ann Transl Med. 2017:5:342

13. Li Y, Zhao Y, Feng L, Guo R. Comparison of the prognostic values of inflammation markers in patients with acute pancreatitis: a retrospective cohort study. BMJ Open. 2017;7:e013206.

14. Ye JF, Zhao YX, Ju J, Wang W. Building and verifying a severity prediction model of acute pancreatitis (AP) based on BISAP, MEWS and routine test indexes. Clin Res Hepatol Gastroenterol. 2017;41:585-91.

15. Wang QM, Luo MW, Xiao B. Value of red blood cell distribution width in assessing the severity of acute pancreatitis. Nan Fang Yi Ke Da Xue Xue Bao. 2017:37:993-6.

16. Goyal H, Gupta S, Singla U. Level of red cell distribution width is affected by various factors. Clin Chem Lab Med. 2016;54:e387.

17. Gülen B, Sonmez E, Yaylaci S, Serinken M, Eken C, Dur A, et al. Effect of harmless acute pancreatitis score, red cell distribution width and neutrophil/lymphocyte ratio on the mortality of patients with nontraumatic acute pancreatitis at the emergency department. World J Emerg Med. 2015; 6:29-33.

18. Guo ZH, Hao JY. The review of acute pancreatitis scoring system. Chin J Clin Hepatol. 2011;27:1170-3. [in Chinese]

19. Ranson JH, Rifkind KM, Roses DF, Fink SD, Eng K, Localio SA. Objective early identification of severe acute pancreatitis. Am J Gastroenterol. 1974;61:443-51.

20. Park JY, Jeon TJ, Ha TH, Hwang JT, Sinn DH, Oh TH, et al. Bedside index for severity in acute pancreatitis: comparison with other scoring systems in predicting severity and organ failure. Hepatobiliary Pancreat Dis Int. 2013;12: 645-50.

21. Gutiérrez-Jiménez AA, Castro-Jiménez E, Lagunes-Córdoba R. Total serum calcium and corrected calcium as severity predictors in acute pancreatitis. Rev Gastroenterol Mex. 2014;79:13-21.

22. Kiliç MÖ, Çelik C, Yüksel C, Yildiz BD, Tez M. Correlation between Ranson score and red cell distribution width in acute pancreatitis. Ulus Travma Acil Cerrahi Derg. 2017;23:112-6.

23. Valverde-López F, Matas-Cobos AM, Alegría-Motte C, Jiménez-Rosales R, Úbeda-Muñoz M, Redondo-Cerezo E. BISAP, RANSON, lactate and others biomarkers in prediction of severe acute pancreatitis in a European cohort. J Gastroenterol Hepatol. 2017;32:1649-56.

24. Cetinkaya E, Senol K, Saylam B, Tez M. Red cell distribution width to platelet ratio: new and promising prognostic marker in acute pancreatitis. World J Gastroenterol. 2014;21:14450-4.

25. Fan ST, Lai EC, Mok FP, Lo CM, Zheng SS, Wong J. Prediction of the severity of acute pancreatitis. Am J Surg. 1993;166:262-8.

26. Li S, Zhang Y, Li M, Xie C, Wu H. Serum albumin, a good indicator of persistent organ failure in acute pancreatitis. BMC Gastroenterol. 2017;17:59.

27. Tefferi A. Anemia in adults: a contemporary approach to diagnosis. Mayo Clin Proc. 2003;78:1274-80.

28. Ghaffari S. Oxidative stress in the regulation of normal and neoplastic hematopoiesis. Antioxid Redox Signal. 2008;10:1923-40.

Ready to submit your research? Choose BMC and benefit from:

- fast, convenient online submission

- thorough peer review by experienced researchers in your field

- rapid publication on acceptance

- support for research data, including large and complex data types

- gold Open Access which fosters wider collaboration and increased citations

- maximum visibility for your research: over $100 \mathrm{M}$ website views per year

At BMC, research is always in progress.

Learn more biomedcentral.com/submissions 